\title{
4.
}

\section{DiGRA Chapter Diversity}

\author{
Hanna Wirman
}

Transactions of the Digital Games Research Association

August 2018, Vol 4 No 1, pp 105-117

ISSN 2328-9422

(C) The text of this work is licensed under a Creative Commons Attribution -- NonCommercial --NonDerivative 4.0 License (http://creativecommons.org/licenses/by-nc-nd/ 2.5/).

IMAGES: All images appearing in this work are property of the respective copyright owners, and are not released into the Creative Commons. The respective owners reserve all rights.

\section{ABSTRACT}

This paper focuses on the perceived value and importance of the regional chapters of Digital Games Research Association (DiGRA). Drawing on a survey conducted among chapter representatives, the viewpoints of nine regional chapter representatives are discussed, particularly from the point of view of how they contribute to better diversity and inclusiveness in such a large international organization. It answers two questions: "What are the advantages of having regional chapters?" and "What exactly constitutes 'regional' in the context of DiGRA chapters?” 
In so doing, the paper establishes regional chapters as important junctures in catering for a wide audience beyond those able and interested in participating in annual DiGRA conferences or partaking in other international events.

Keywords

DiGRA, association, regional, ethnic, chapters, local, international, global

\section{INTRODUCTION}

Digital Games Research Association (DiGRA) currently supports 11 regional chapters on four continents. Chapters operate autonomously and in a self- sustaining manner, yet in association with the main DiGRA following the core principles, values, and objectives of the association. Among others, regional members are encouraged and welcomed to participate in annual DiGRA conferences. From the point of view of their establishment, the DiGRA executive board is responsible for vetting and approving chapter proposals. To help sustain its relationship with local chapters as well as to facilitate and support their local activities, DiGRA has named a designated International Chapter Officer within its executive board since 2012 .

Written from the perspective of how DiGRA promotes diversity through its chapter structure, this paper has two goals. The first is to introduce DiGRA chapter structure and the developments thereof during the past four years. I will specifically elaborate some of the differences between local chapters and consider their regionally relevant contributions. The second aim of the paper is to draw examples from various chapters in order to look into what are some of the benefits and challenges that the chapters have in respect to contributing to DiGRA diversity and inclusiveness.

Material for this study was collected by myself during my term as the DiGRA Chapter Officer through an online survey sent to the 
primary contact persons of each regional chapter during spring and summer of 2017. The survey focused on examining the perceived and practical relationship between regional chapters and the main DiGRA organization. Questions were asked about regional activities and chapter members' abilities and interests to participate in international DiGRA conferences. Furthermore, the survey aimed at understanding the value regional bodies of games researchers find in being part of a larger, international entity.

Nine out of eleven chapter representatives answered the survey, including the following DiGRA chapters: Australian, Chinese, Finnish, Israeli, Italian, Japanese and British DiGRA as well as D-A-CH DiGRA (chapter for German-speaking countries) and DiGRA Flanders. Dutch and Turkish DiGRA chapter representatives were not available to participate in the study. Additionally, to support articulation of the value of regional chapters in respect to participation in international DiGRA conferences, country-based participation figures of annual DiGRA conferences were obtained from the organizing committees of DiGRA 2016 and DiGRA 2017.

The paper will conclude by suggesting new ways to support and help sustain regional chapters of DiGRA. Regions where local chapters do not operate are compared with the respective regions of origin of DiGRA conference participants in the past years. Ultimately, this paper sheds light on the immense value of regional organizations and the meaningful differences between them. Since I personally acted as the first president of Chinese DiGRA, examples drawn from this chapter are relatively frequent in what follows.

\section{CHAPTER OPERATIONS}

Regional DiGRA chapter establishment seems to follow two main patterns. Most commonly, DiGRA chapters have been organized in regions where a strong community of active game researchers familiar with DiGRA seek more frequent sharing compared to 
what an annual conference can provide. These chapters typically consist of active DiGRA goers and well-sustained research centers that host several games researchers. Alternatively, the initiation of the Chinese DiGRA chapter serves as an example of where a chapter is set up to bring together scattered individuals researching games through the introduction of this international organization. In the latter case, DiGRA has helped to legitimize the study of games and provided individuals with a coordinated and institutionally recognized community solely focused on games, where little or none existed prior to the chapter. Here, DiGRA lends the chapter the credibility of studying games in the first place.

As the organization grows and research in the field becomes more competitive, regional DiGRA chapters are increasingly valuable in creating avenues for supporting games research regardless of geographic or linguistic differences. As an example, DiGRA Japan organizes several events every year for around 300 chapter members. However, only a fraction of them ever attend the main DiGRA conference. Similarly, students at all levels often rely on local chapters, as the cost of participating in international events hinders contribution and attendance. Moreover, local chapters have strong and sustained partnerships with representatives of local industries. While engagement with the games industry is in DiGRA's interest, such collaboration typically best takes place at the regional level.

The activities of regional chapters vary from chapter to chapter, most focusing on maintaining local communities through organizing events, and fostering and promoting games research (and education) by providing support and research that directly addresses local issues and interests. The main activity of regional chapters is an annual conference. Five out of nine chapters included in the survey organize a conference every year. Twothirds of the chapters organize other talks and seminars, and onethird casual gatherings. Local chapters also support initiating collaboration between members, in sharing resources, and in 
supporting other events. Individual chapters adjust their operational models from time to time. It is not unusual for a chapter to remain dormant for several months or even years due to member inactivity. However, given the lack of strong regulations on how chapters can or should be run, relaunching chapters becomes relatively straightforward.

Regional DiGRA chapters are also typically very dynamic and responsive to new research foci and local interests. Alongside supporting regional DiGRA sub-communities, and therefore fostering regional diversity of DiGRA, local chapters promptly tackle local issues and interests that are beyond the main DiGRA's reach. Regional chapters foster diversity by addressing topics that may appear marginal with respect to the main DiGRA and that need urgent attention.

\section{Language and regionality}

Language plays a major part in running regional chapters and in respect to chapter members' involvement in international DiGRA events. Survey participants suggest that the language barrier or an interest to operate using the local language is one of the main reasons for setting up and framing a chapter. Among others, DiGRA for German-speaking countries is specifically organized around the German language.

The ability to communicate in English, which is the language used at DiGRA annual conferences, influences chapter members' participation in international events. Typically, chapter members from English-speaking countries (i.e. British DiGRA and DiGRA Australia) as well as from many European countries and regions are active in participating in DiGRA annual conferences. Meanwhile, members from Chinese DiGRA, DiGRA Japan, and Israeli DiGRA show significantly less participation in international conferences. In particular, the largest chapter, Japanese DiGRA, relies heavily on the Japanese language, and members are less likely to participate in DiGRA activities outside 
of Japan. It has also been clear since the chapter's initiation in 2014 that only a small number of scholars in the Chinese chapter have actively participated in international DiGRA conferences. However, the reason could be the geographical distance and mismatch in academic calendars, which responses suggest. Ph.D. students, meanwhile, are less likely to have the funds to travel to, and benefit from, regional events.

The participation figures of DiGRA 2016 (Chart 1) and DiGRA 2017 (Chart 2) serve to illustrate how annual DiGRA conference participation is skewed towards English-speaking and European countries regardless of the actively operating chapters elsewhere, particularly in Asia. One practical solution to reduce segregation among researchers could be to translate research from regional chapter conferences, and include these in the DiGRA Digital Library (as in the case of the Chinese DiGRA 2016 Conference).

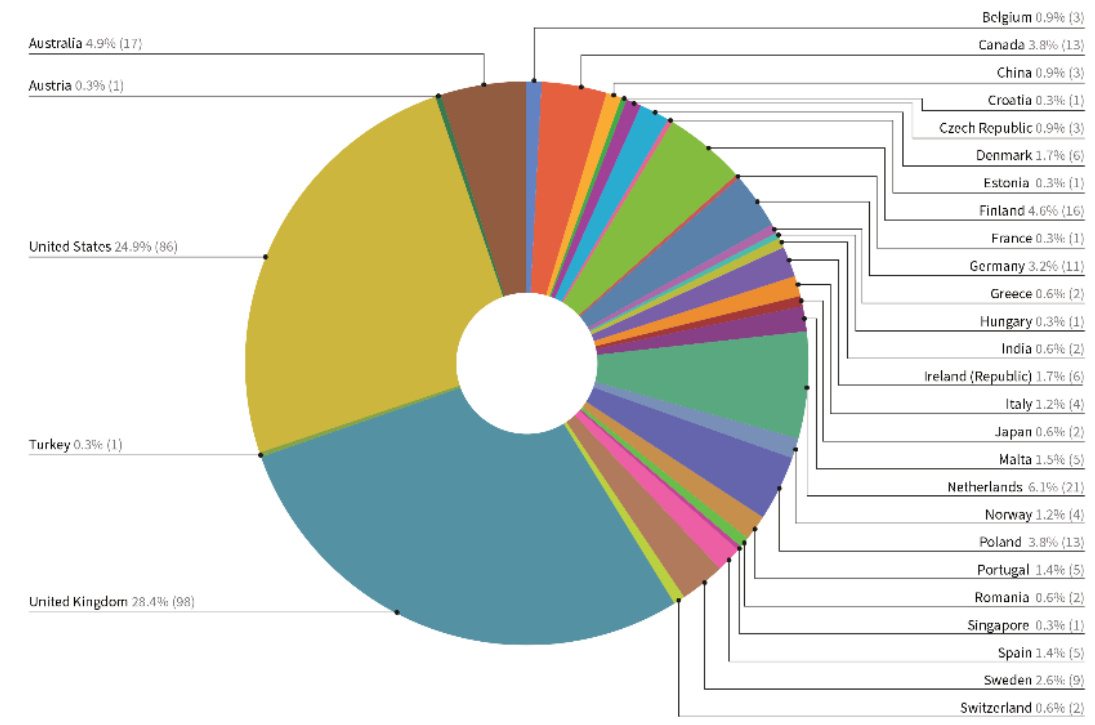

Chart 1: Conference participation demographics: Dundee, UK, 2016 


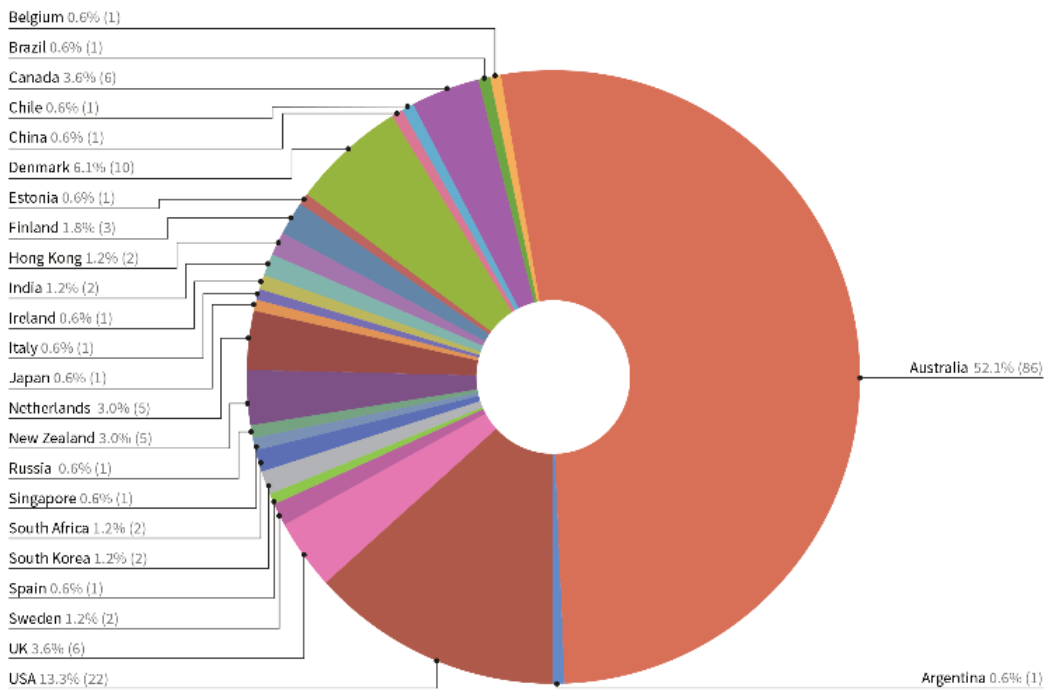

Chart 2: Conference participation demographics: Melbourne, Australia, 2017

\section{'Regional' in DiGRA chapters}

Alongside language, what makes DiGRA chapters regional is primarily related to geographical proximity and to an interest in topics specific to that area. For instance, while many of the concerns, opportunities and discussions among Chinese DiGRA members bear a close similarity to those that colleagues in other regions have experienced in the past, the Chinese regional context has its unique characteristics. Among others, the regional games industry has seen an extremely rapid and extensive growth in terms of both numbers of players and revenue. As the Western news media has addressed the Chinese context through extremities and curiosities (Wirman 2015), the current relatively stabilized game markets and largest player communities in the world provide a fruitful object for groundbreaking game studies beyond anecdotal reference points. Like Liboriussen and Martin (2016, n.p.) outline, regional games research "attends to local places and cultures but also, at least to some extent, to how the local substance connects 
with higher-order economic, cultural and political structures.” Regional chapters thus dig deeper into regional matters given the shared background knowledge and pre-existing ability to contextualize complex regional matters. Regional chapters may suggest a more 'diverse' DiGRA, yet the scholarly practices of attuning to a variety of topics and regional interests are perhaps of more importance: "although the significance of diversity can be described as international, the means by which diversity manifests itself will be local.” (Ahmed 2012) Accordingly, the chapters contribute to the idea of advocating local manifestations for 'doing diversity’ (Ibid.).

Regional matters often also touch on issues of politics and power. Among others, the Chinese government's forceful regulation of game content, and restrictions related to the access of non-Chinese games and game studios to the local market as well as existing diverse practices of monetization and publishing are without direct parallels in the Western world, and therefore call for locally attuned, highly context-aware game studies. Here we may assume that regional game studies are possibly more closely interlinked with untangling complicated issues and nuances than those typically presented at international DiGRA conferences. At the very least, such topics can be addressed at a deeper level when event participants can expect to be informed of current, local affairs.

The intricacies of local issues are only apparent to those personally witnessing and experiencing such events. Paraphrasing Dei (2013) and drawing on an example from the African context, Gumbo (2014) suggests that Global South's academic interests, for example, are often intertwined with political activism: "in the African scholar's attempt to negotiate co-existence of western and indigenous research, western academy constantly asks the African scholar to separate his scholarship from his political activism.” In non-democratic and less economically developed countries, local games studies are necessarily also always closely influenced by the everyday struggles on the political and economic plane. Regional 
chapters thus offer the main DiGRA organization possibly a fuller picture of how games situate as part of people's everyday lives and local culture. Gumbo has further stressed the importance of benefiting the local community through research: "questioning the hegemonic attitudes that treat the researched indigenous communities as though they were unthinking tanks from which to pour out research data without involving them actively and doing one's homework about how the research will benefit them.” (Gumbo 2014, 558) Regional game studies are potentially more informed and interested in serving the local interests than those studying them from outside of the region.

In respect to regionally specific interests, regional chapters have the potential to contribute to having a wider selection of games under scholarly scrutiny. This would then help to alleviate the lack of diversity in the games we study - or the 'game citation paradox' - where "a majority of papers are focused on a minority of games." (Coavoux, Boutet and Zabban, 2017) ${ }^{1}$

The games industry is another area of interest for games scholars that has regional specificities which are difficult to translate to a greater community. While regional chapters gather scholars in the same geographical area, they also invite local members from the industry. Eight out of nine chapters covered in the survey describe that they collaborate with the local industry, with some having a chapter board member from the commercial world, and many of them inviting speakers and event participants from the industry. The themes of regional DiGRA conferences reflect an interest to work closely with the industry, such as "Decoding the Academic-Industrial-Gameplay Complex.” (Chinese DiGRA 2016) Understandably, participants who approach regional chapters from the industry are particularly focused on local aspects of game studies and interested in the direct benefits that the study of games can bring to the field of designing, developing and

1. The study by Coavoux, Boutet and Zabban (2017) focuses on research published in

English. It is likely that the results would be significantly different if other languages would be covered, too. 
marketing games. Many chapter representatives agree that close communication with the local industry is an important aspect of the operations of the regional chapter. For DiGRA as a whole, chapters, therefore, provide an extension from academia to the industry.

Regardless of the value in 'going local', all except one of the studied DiGRA regional chapters agree that 'being part of a larger community' is one of the main reasons for having a regional chapter. Similarly, chapter representatives believe that the DiGRA name helps in being treated more seriously in the local context. Almost as important for the regional chapters is that being part of DiGRA offers them a point of comparison, a tool to understand their own contribution to games research. A convenient mechanism for inviting speakers from other regions, support for a mailing list, legitimization in the eyes of the local industry, and adoption of DiGRA operational models are all important for at least three of the chapters. The regional and the global benefits, therefore, support each other and depend on each other.

\section{WHOSE DiGRA?}

Figure 1 shows the presence of DiGRA chapters in different regions (red circles) and the DiGRA 2017 conference participants' countries of origin. Accordingly, current chapters are disproportionately covering Europe and Asia, even though a significant number of active DiGRA conference participants come from North America. Assuming a continuous increase in people's interest to make, play and study games, DiGRA chapters are likely to be established by emerging game studies communities in the coming years. There is currently an interest to start DiGRA Latin America, for example. 


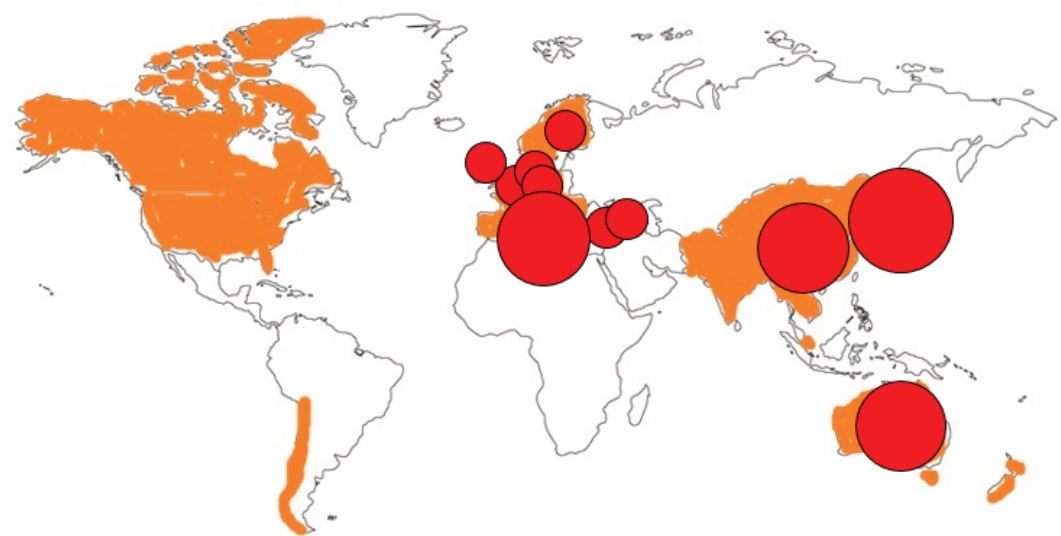

Figure 1: DiGRA chapters (red) and the countries of origin of DiGRA 2017 conference participants (orange) mapped.

However, establishing a chapter of an international, European and North American centered organization outside of these regions brings with it an existing history of doing, seeing and understanding things, or in this case, games. In building networks and supporting local research, Chinese DiGRA has aimed at being particularly sensitive in providing a platform that does not advocate a specific hegemonic Western canon of game studies. However, many of the founding members and members of the current Chinese DiGRA have been educated in Europe. Furthermore, English has been one of the languages used at the conferences, given the diverse backgrounds of delegates and the lack of a single shared language (and the political sensitivity of selecting only one among the few available). Early attempts at dividing conference papers into a Chinese language track and an English language track almost completely negated the original interest behind the chapter as an entity that brings scholars together. Yet simultaneous interpretation at conferences and other events is a significant cost consideration for small, regional organizations. 
Moreover, there is no easy way for regional DiGRA events to function as multilingual platforms in which international participants can conveniently participate. However, the full adoption of the existing Western canon of game studies literature or the application of DiGRA operational models can locally threaten to impose non-fitting practices within regional communities: "When we as scholars unthinkingly adopt the discourse and knowledge of mainstream Euro-American organizational communication scholarship, we potentially absorb, without reflection, a particular way of understanding the world.” (Broadfoot and Munshi, 2007)

The future challenge of DiGRA as an international organization is, as I see it, in how it deals with regional diversification and whether it becomes a project of colonization or decolonization. It may be relatively unique to DiGRA as an academic organization to have such regional chapters that operate independently without members' active participation in international activities. There exist isolated local nuances in 'doing game studies' under the large international umbrella. How, then, can these regional interests and local canons be brought to the international stage without misinterpretation, misrepresentation and misuse? How much do the regionally active scholars need to know about ways of 'doing game studies' outside of their region before entering venues like the annual international conferences? After all, solely Englishspeaking scholars already miss bodies of valuable work, unable to search or read existing literature in other languages (particularly when it relates to a specific cultural/geographic context).

Finally, we may look at regions such as Poland, where game scholarship is particularly active and internationally well represented, yet no local DiGRA chapter has been initiated. Several South American countries and Russia also contribute to the growing knowledge in the area of games research, making their absence on the map of Image 1striking. Further study is required to understand the actual contributions from these areas and to benefit from the already existing scholarly knowledge of games 
in these regions. Explicating these regions' contribution within or outside of DiGRA would further help in understanding how 'diverse' DiGRA research actually is and how big a part chapters play in it.

\section{BIBLIOGRAPHY}

Broadfoot, K. J. and Munshi, D. Diverse Voices and Alternative Rationalities: Imagined Forms of Postcolonial Organizational Communication. Management Communication Quarterly, 21, 249-267, 2007.

Coavoux, S., Boutet, M. and Zabban, V. What We Know About Games. Games and Culture, 12(6), 563-584, 2017.

Dei, G. J. S.Critical perspectives on indigenous research.Socialist Studies, 9(1), 27-38, 2013.

Gumbo, M.T. Symbolic Xenophobia Mirrored through the Struggle of an African Scholar in the Academic Space. Mediterranean Journal of Social Sciences, 5(1), 2014.

Liboriussen, B. and Martin, P. Regional Game Studies. Game Studies, 16(1), 2016.

Wirman, H. "Sinological-Orientalism in Western News Media: Caricatures of Games Culture and Business". Games and Culture11(3), 298-315, 2015. 